\title{
Analysis of medical service use of knee osteoarthritis and knee meniscal and ligament injuries in Korea: a cross-sectional study of national patient sample data
}

Chang Yong Suh ${ }^{1 \dagger}$, Yoon Jae Lee ${ }^{1 \dagger}$, Joon-Shik Shin ${ }^{1}$, Jinho Lee ${ }^{1}$, Me-riong Kim², Wonil Koh ${ }^{1}$, Yun-Yeop Cha ${ }^{3}$, Byung-Cheul Shin ${ }^{4,5}$, Eui-Hyoung Hwang ${ }^{4,5}$, Kristin Suhr ${ }^{6}$, Mia Kim and In-Hyuk Ha ${ }^{7 *}$

\begin{abstract}
Background: Osteoarthritis $(\mathrm{OA})$ and meniscal and ligament injuries of the knee are the two most common knee disorders in Korea. The aim of this study was to analyze the demographic characteristics, medical service use and related costs for these disorders, and the results are expected to help inform practitioners, researchers, and policy-makers.

Methods: The present study aimed to evaluate incidence and patient characteristics, and to assess current medical service use, usual care, and medical expenses of knee disorders by analyzing 2014 national patient sample data from the Korean Health Insurance Review and Assessment Service. Data was extracted using 3\% stratified sampling from all Korea national health insurance claims submitted in 2014, and analyzed. Usual care for M17 knee osteoarthritis and S83 knee meniscal and ligament injury codes of the International Classification of Diseases, 10th revision (ICD-10) were determined by investigating total number of patients, sociodemographic characteristics, days in care, number of visits, and expenses.

Results: Knee OA showed the highest incidence in females aged $\geq 60$ years, whereas meniscal and ligament injuries of the knee were most prevalent among patients aged $<20$ years and young adults. Total inpatient care expenses exceeded the cost of ambulatory care for both disorders. Ambulatory care was mainly provided at primary care clinics, with $90 \%$ of these visits made to orthopedic specialists. Medical expenses for knee OA and meniscal and ligament injuries were largely due to procedures/surgeries and injections, and procedures/surgeries and hospitalizations, respectively. Total replacement arthroplasty was the most commonly performed surgery for knee OA, while meniscectomy and cruciate ligament reconstruction were the most often performed surgeries for meniscal and ligament injuries. Intra-articular injection rates were 55\% in knee OA patients and 3\% in meniscal and ligament injury patients. Aceclofenac, diclofenac, and tramadol were the most frequently prescribed analgesics.
\end{abstract}

Conclusions: The current findings may be used as basic data for establishing medical policies and can benefit researchers and clinicians in recognizing trends and patterns of treatment for knee disorders.

Keywords: Knee osteoarthritis, Knee meniscal and ligament injury, Medical service use, Usual care, Korean Health Insurance Review and Assessment Service-National Patient Sample (HIRA-NPS) data

\footnotetext{
* Correspondence: hanihata@gmail.com

${ }^{\dagger}$ Equal contributors

1Jaseng Spine and Joint Research Institute, Jaseng Medical Foundation, 858

Eonju-ro, Gangnam-gu, Seoul, Republic of Korea

Full list of author information is available at the end of the article
} 


\section{Background}

Knee osteoarthritis (OA) is one of the most common disorders in the U.S., and symptomatic OA prevalence was found to be as high as $10 \%$ in men and 13\% in women aged $\geq 60$ [1]. Similarly, symptomatic knee OA was shown to affect $9.3 \%$ and $28.5 \%$ of Korean men and women aged $\geq 50$, respectively, with a steep increase in prevalence in older populations [2]. Meanwhile, knee meniscal injury patients tend to be younger than knee OA patients, and incidence was reported as $3-5 \%$ in the U.S. [3] and 10.6\% in Korean populations [4]. According to the U.S. Medical Expenditure Panel Survey, insurance coverage and out-of-pocket expenses for knee OA amounted to 185 billion U.S. dollars, denoting its significant socioeconomic impact [5]. In addition, arthroscopic partial meniscectomy is one of the most frequently performed orthopedic surgeries in the U.S., with 700,000 new cases performed each year, and estimates for direct annual medical costs were put at 4 billion U.S. dollars [6].

The epidemiology and pathology of knee OA and meniscal injury differ substantially regarding their onset, age, and etiology. Population-wide studies have purported that incidence estimates of knee meniscal injury are highest in adolescent and young men [7], stating that these populations are at higher risk of injury as they are more likely to engage in competitive sports such as ball games, while older adults are at lower risk of injury as they are more likely to participate in non-competitive sports such as walking, jogging and swimming [8]. On the other hand, knee OA prevalence increases rapidly after the age of 50, which is especially pronounced in post-menopausal women due to the effect of hormonal imbalance on cartilaginous tissue [9]. Although the epidemiology of knee osteoarthritis and injury varies considerably, their etiology is often viewed to share certain traits; injury of the anterior cruciate ligament (ACL) or the meniscus may incur joint instability and damage the cartilage surface, leading to chronic disability and potential knee OA $[10,11]$.

The National Health Insurance Service (NHIS) in Korea covers 47 million out of 51 million South Korean residents. Claims data are generated when a medical institution provides medical services to a patient and applies for reimbursement from the NHIS, and therefore contain information on the patient and medical institution, a complete list of insured medical services that were provided (e.g., treatment, examinations, and prescriptions), and their related costs. The two highest frequency knee disorders, knee OA and knee meniscal and ligament injuries, were comprehensively analyzed for patient characteristics and expenditure (i.e., surgery, hospitalization, physical therapy and medication costs) to the aim of providing basic information to policymakers and practitioners in Korea. Moreover, given that standard care based on evidence and the usual care provided in real world settings frequently digress and that by country and culture, the implications of current reports on high-frequency medical service use (i.e., pharmacological, nonpharmacological, and surgical interventions) hold relevance at an international level also through illustration of concurrent clinical practice in knee disorders.

The aim of this study was to provide preliminary data towards establishing a basic guideline for general medical care based on the prevalence, current use of medical services, and costs of knee OA and meniscal and ligament injuries from the 2014 claims data submitted to the Korea Health Insurance Review and Assessment Service (HIRA). This analysis may be used to assist policymakers, practitioners, and researchers by providing a window into the most common treatments for knee conditions.

\section{Methods \\ Data and subjects}

The 2014 Health Insurance Review and Assessment Service-National Patient Sample (HIRA-NPS) data were analyzed. Claims data are generated when medical institutions apply for reimbursement of medical costs partially covered by Korea National Health Insurance. The data include medical record details (e.g., treatments, procedures, examinations, and prescriptions), diagnosis codes, co-payment paid by the patient and the insurance benefit paid by Korea National Health Insurance, patient demographics (e.g., age and sex), and information on the service provider (medical institution).

Provided yearly for research purposes, the HIRA-NPS datasets are extracted from the raw claims database using random sampling, stratified according to age groups and sex, with removal of identifying personal and institutional information. Each dataset consists of claims records for the corresponding year. Regardless of inpatient/outpatient status, $3 \%$ of the total patient population for each year was sampled and extracted, which approximates to 1.4 million patients. As each individual patient is coded with a unique identifier which is randomly generated and not privacy-sensitive, multiple visits of individual patients are easily traceable [12]. The current study chose to analyze the total number of patients by treatment type as opposed to total number of visits to the aim of illustrating individual medical service use in this patient population. Upon analysis, the results were presented both as inpatient and outpatient groups separately, and in total. The number of in- and outpatients indicate the number of patients who were hospitalized and those who visited the outpatient department one or more times for knee disorder-related diagnosis codes during the corresponding year, respectively. Multiple hospitalizations or outpatient visits of individual 
patients were viewed as duplicates and disregarded. Total counts for medical services were similarly tallied as the number of patients who used each respective service and not the number of treatment sessions. The actual parameters of the South Korean population may therefore be estimated from the current sample data statistics by multiplying the weighting value of 33.3 as the database is generated from $3 \%$ stratified sampling of total patients. In the present study, the statistical weight was not applied as the weighting was identical across all data and therefore did not affect the interpretation of results.

\section{Knee $O A$ and knee meniscal and ligament injuries}

Knee disorders were classified as M17 (arthrosis of the knee), S83 (dislocation, sprain, and strain of joints and ligaments of the knee), M22 (disorders of the patella) and M23 (internal derangement of the knee), according to the International Statistical Classification of Diseases and Related Health Problems, 10th revision (ICD-10). The number of individuals with knee disorder codes and radiological imaging of the knee were 48,321 with M17, 3087 with M22, 7103 with M23, and 19,136 with S83 in the 2014 dataset (Additional file 1: Table S1). Only those coded as M17 and S83 were included in this study as relatively few individuals were diagnosed with M22 and M23. A total of 48,000 outpatients and 3084 inpatients were diagnosed and treated under M17, and 18,540 outpatients and 2434 inpatients were diagnosed and treated under S83. Duplicate cases existed when a patient received both ambulatory and hospital care under the same code; thus, the total number of patients was smaller than the numerical sum of inpatients and outpatients. Through literature review and author discussion, M17 was diagnosed as knee OA [13-15] and S83 as knee meniscal and ligament injury [16].

\section{Analysis}

The demographic characteristics of knee disorder patients as classified using ICD-10 diagnostic codes were examined. Age groups were divided into 10-year intervals: less than 20 years, $20-29$ years, $30-39$ years, and so on. Each patient was also classified by type of insurance as being eligible for either National Health Insurance or Medicaid. Medical institutions were categorized as primary care clinics, hospitals, general hospitals, tertiary hospitals, long-term care hospitals, or public health centers. The specialty of the attending physician was also assessed. The total treatment expenses were divided and analyzed according to the medical service codes designated by the Korean Ministry of Health and Welfare (i.e., costs per visit (consultation), hospitalization, medication, injection, anesthesia, physiotherapy, psychotherapy, procedure/surgery, examination, and radiographic evaluation/intervention). Total expense was defined as the total costs incurred for healthcare of the insured patient at medical institutions, which equaled the sum of benefit paid by the National Health Insurance Service and co-payment expenses paid by patients, of which the reimbursement amount was reviewed and determined by HIRA.

Details on the use of surgeries, injections, physiotherapy, and analgesics were investigated according to the corresponding code count; the relevant codes for these services are summarized in Additional file 2: Table S2. When assessing surgical patterns, the procedure codes were excluded from the procedure/surgery analysis because surgery codes were always accompanied by collateral procedure codes. Surgery codes that are not limited to the knee such as joint excision, osteochondral autograft transplantation, osteotomy and internal fixation, and subcutaneous tenotomy were also excluded.

Injection use was investigated according to the administration route, with intra-articular, subcutaneous, and intramuscular injections being of particular interest. Although intravenous, continuous intravenous, and perineural injections were often administered for knee disorders in clinical settings, the authors concluded that these injection types were generally not directly related to knee disorder treatment or for anti-nociceptive purposes, and these codes were accordingly excluded.

The most frequently applied physiotherapies are arranged in order of their prescription frequencies. Analgesics prescribed for inpatient and outpatient use were classified in order of prescription frequency, according to the 5th Anatomical Therapeutic Chemical (ATC) Classification System levels. Narcotic and non-narcotic substances were categorized as previously reported by the Korean National Evidence-based Health Care Collaborating Agency [17]. ATC codes were developed by the WHO Collaborating Centre for Drug Statistics Methodology in 1976 [18]. The 1st, 2nd, 3rd, 4th, and 5th ATC code levels indicate the anatomical target groups, therapeutic groups, therapeutic/pharmacologic subgroups, chemical/therapeutic/pharmacologic subgroups, and chemical substances, respectively, for the systematic classification of drug substances [19].

Most 5th ATC level drug substances have corresponding chemical names, with a few exceptions such as acetaminophen and paracetamol. Although this study used 5th ATC level terms to standardize nomenclature, four analgesics (7 in Additional file 3: Table S3) did not have corresponding 5th level codes, and in those instances, chemical names were used instead. Drug prescriptions were identified using the relevant claims data, regardless of dose.

\section{Statistical analysis}

The demographic characteristics and medical details regarding surgeries, injections, physiotherapy, and drug 
prescriptions of patients with knee OA and meniscal and ligament injury are presented as frequencies and percentages (\%) following frequency analyses. Patient percentages were determined using the total number of patients in each relevant diagnosis group as the denominator, and medical expense percentages were similarly calculated for total expenses, per-patient expenses, and costs per service using the total treatment expense for knee OA and knee meniscal and ligament injury as the denominator, respectively. Statistical analyses were performed using SAS, ver. 9.3 package (SAS Institute Inc., Cary, NC, USA).

\section{Results}

\section{Current use of medical treatment for knee OA and knee meniscal and ligament injuries}

The total number of patients, total expenses, per-patient expenses, average days of care, and average number of visits were higher for patients with knee OA than for those with knee meniscal and ligament injuries, regardless of whether the patients were treated as inpatients or outpatients. Although the total number of inpatients was smaller than the total number of outpatients, the total expenses for inpatients were higher in both types of knee disorders. The per-patient expense for inpatients was approximately 20 times higher than that for outpatients, irrespective of the type of knee disorder. However, the average number of days of care and number of visits were more than three times higher for hospitalized patients than for those receiving ambulatory care (Table 1).

\section{Sociodemographic characteristics of knee $O A$ and knee meniscal and ligament injury patients}

The majority of patients with OA of the knee and knee meniscal and ligament injury received ambulatory care with approximately $6 \%$ of knee OA patients and $12 \%$ of meniscal and ligament injury patients hospitalized. The incidence of knee OA was positively correlated with age, showing a steep increase after the age of 50 years. However, the number of knee meniscal and ligament injury patients was highest in age $<20$ years, with the numbers remaining constant from age 20 to 60 years, and declining thereafter. The age distribution was similar for both inpatients and outpatients with knee OA. On the other hand, the percentage of knee meniscal and ligament injury in patients aged $<20$ years who were hospitalized was approximately half the number of those receiving outpatient care. Knee OA was found to be more prevalent in women than in men, as was clearly evidenced by the fact that $82 \%$ percent of inpatients were women. On the contrary, the incidence of knee meniscal and ligament injuries was slightly higher in men than in women.

Almost all knee disorder patients were covered by $\mathrm{Na}$ tional Health Insurance, and the treatment patterns did not differ substantially between inpatients and outpatients. About $70 \%$ of all patients visited primary care clinics, while the rest visited hospitals, general hospitals, and tertiary hospitals (listed in order of decreasing frequency of use). Notably, more than half of all knee OA and meniscal and ligament injury inpatients received medical care in hospitals instead of more accessible primary care clinics, which differed from the pattern seen in outpatients, and the percentage of general, tertiary, and long-term care hospitals increased substantially in inpatients. However, overall usage of medical institutions did not differ greatly between knee OA and meniscal and ligament injury patients. Approximately $90 \%$ of all patients, regardless of the type of disorder or whether they were receiving inpatient or outpatient care, sought medical care from an orthopedics department. Apart from orthopedics, knee OA patients were treated in anesthesiology, general surgery, and neurosurgery departments, and knee meniscal and ligament injury patients were treated in general surgery, neurosurgery, and emergency medicine (EM) departments. Other than orthopedics, inpatients were often treated in specialty departments such as rehabilitative medicine and general surgery, whereas radiology, EM, and general practice departments were utilized for outpatients (Table 2).

\section{Distribution of medical expenses in knee OA and knee meniscal and ligament injuries}

Medical expenses for knee OA and knee meniscal and ligament injury cases were classified into 10 categories:

Table 1 General medical service use and expenses for knee osteoarthritis and knee meniscal and ligament injury in Korea

\begin{tabular}{|c|c|c|c|c|c|c|}
\hline Visit type & Diagnostic groups & Number of patients & Total expense ${ }^{a}$ & Expense-per-patient ${ }^{a}$ & Days of treatment ${ }^{b}$ & Number of visits ${ }^{c}$ \\
\hline \multirow[t]{2}{*}{ Total } & Knee OA & 48,321 & $24,526,738,820$ & $507,579.3$ & 11.0 & 9.1 \\
\hline & Knee meniscal and ligament injury & 19,136 & $5,800,760,680$ & $303,133.4$ & 5.8 & 4.7 \\
\hline \multirow[t]{2}{*}{ Outpatients } & Knee OA & 48,000 & $10,758,908,490$ & $224,143.9$ & 8.7 & 7.8 \\
\hline & Knee meniscal and ligament injury & 18,540 & $1,660,439,710$ & $89,559.9$ & 3.4 & 3.2 \\
\hline \multirow[t]{2}{*}{ Inpatients } & Knee OA & 3084 & $13,767,830,330$ & $4,464,277.0$ & 36.4 & 22.2 \\
\hline & Knee meniscal and ligament injury & 2434 & $4,140,320,970$ & $1,701,035.7$ & 20.1 & 12.3 \\
\hline
\end{tabular}


Table 2 Sociodemographic characteristics of knee osteoarthritis and knee meniscal and ligament injury patients

\begin{tabular}{|c|c|c|c|c|c|c|c|c|c|c|c|c|c|}
\hline \multirow[t]{3}{*}{ Characteristics } & & \multicolumn{4}{|l|}{ Total } & \multicolumn{4}{|l|}{ Inpatient } & \multicolumn{4}{|l|}{ Outpatient } \\
\hline & & \multicolumn{2}{|l|}{ Knee OA } & \multicolumn{2}{|c|}{$\begin{array}{l}\text { Knee meniscal } \\
\text { and ligament injury }\end{array}$} & \multicolumn{2}{|l|}{ Knee OA } & \multicolumn{2}{|c|}{$\begin{array}{l}\text { Knee meniscal } \\
\text { and ligament injury } \\
\end{array}$} & \multicolumn{2}{|l|}{ Knee OA } & \multicolumn{2}{|c|}{$\begin{array}{l}\text { Knee meniscal } \\
\text { and ligament injury }\end{array}$} \\
\hline & & $N=48,321$ & $\%$ & $N=19,136$ & $\%$ & $N=3084$ & $\%$ & $N=2434$ & $\%$ & $N=48,000$ & $\%$ & $N=18,540$ & $\%$ \\
\hline \multirow[t]{7}{*}{ Age (years) } & $<20$ & 243 & 0.50 & 4290 & 22.42 & 1 & 0.03 & 272 & 11.18 & 242 & 0.50 & 4252 & 22.93 \\
\hline & $20 \sim 29$ & 457 & 0.95 & 2665 & 13.93 & 11 & 0.36 & 411 & 16.89 & 450 & 0.94 & 2592 & 13.98 \\
\hline & $30 \sim 39$ & 890 & 1.84 & 2488 & 13.00 & 38 & 1.23 & 359 & 14.75 & 877 & 1.83 & 2415 & 13.03 \\
\hline & $40 \sim 49$ & 3812 & 7.89 & 3131 & 16.36 & 199 & 6.45 & 470 & 19.31 & 3758 & 7.83 & 3032 & 16.35 \\
\hline & $50 \sim 59$ & 12,856 & 26.61 & 3542 & 18.51 & 772 & 25.03 & 539 & 22.14 & 12,736 & 26.53 & 3387 & 18.27 \\
\hline & $60 \sim 69$ & 14,790 & 30.61 & 1865 & 9.75 & 960 & 31.13 & 251 & 10.31 & 14,729 & 30.69 & 1777 & 9.58 \\
\hline & $\geq 70$ & 15,273 & 31.61 & 1155 & 6.04 & 1103 & 35.77 & 132 & 5.42 & 15,208 & 31.68 & 1085 & 5.85 \\
\hline \multirow[t]{2}{*}{ Sex } & Male & 13,141 & 27.20 & 10,445 & 54.58 & 546 & 17.70 & 1438 & 59.08 & 13,054 & 27.20 & 10,171 & 54.86 \\
\hline & Female & 35,180 & 72.80 & 8691 & 45.42 & 2538 & 82.30 & 996 & 40.92 & 34,946 & 72.80 & 8369 & 45.14 \\
\hline \multirow[t]{3}{*}{ Insurance type } & $\mathrm{NHI}$ & 45,183 & 93.51 & 18,483 & 96.59 & 2853 & 92.51 & 2356 & 96.80 & 44,879 & 93.50 & 17,912 & 96.61 \\
\hline & $\mathrm{MD}$ & 3234 & 6.69 & 651 & 3.40 & 230 & 7.46 & 74 & 3.04 & 3215 & 6.70 & 625 & 3.37 \\
\hline & VH & 135 & 0.28 & 21 & 0.11 & 11 & 0.36 & 6 & 0.25 & 133 & 0.28 & 19 & 0.10 \\
\hline \multirow{7}{*}{$\begin{array}{l}\text { Medical institution } \\
\text { type }^{b}\end{array}$} & Clinic & 36,731 & 76.01 & 13,343 & 69.73 & 592 & 19.20 & 590 & 24.24 & 36,570 & 76.19 & 13,115 & 70.74 \\
\hline & Hospital & 12,925 & 26.75 & 4814 & 25.16 & 1816 & 58.88 & 1263 & 51.89 & 12,616 & 26.28 & 4452 & 24.01 \\
\hline & $\mathrm{GH}$ & 5371 & 11.12 & 2053 & 10.73 & 646 & 20.95 & 569 & 23.38 & 5251 & 10.94 & 1872 & 10.10 \\
\hline & $\mathrm{TH}$ & 1801 & 3.73 & 437 & 2.28 & 243 & 7.88 & 129 & 5.30 & 1774 & 3.70 & 408 & 2.20 \\
\hline & $\mathrm{LCH}$ & 621 & 1.29 & 85 & 0.44 & 176 & 5.71 & 25 & 1.03 & 457 & 0.95 & 65 & 0.35 \\
\hline & $\mathrm{PHC}$ & 407 & 0.84 & 21 & 0.11 & 2 & 0.06 & - & - & 407 & 0.85 & 21 & 0.11 \\
\hline & $\mathrm{KMH}$ & 126 & 0.26 & 59 & 0.31 & 80 & 2.59 & 34 & 1.40 & 50 & 0.10 & 26 & 0.14 \\
\hline \multirow[t]{11}{*}{ Medical specialty ${ }^{c}$} & OS & 43,654 & 90.34 & 17,056 & 89.13 & 2852 & 92.48 & 2304 & 94.66 & 43,379 & 90.37 & 16,486 & 88.92 \\
\hline & AN & 3108 & 6.43 & 300 & 1.57 & 32 & 1.04 & 11 & 0.45 & 3083 & 6.42 & 293 & 1.58 \\
\hline & GS & 2350 & 4.86 & 588 & 3.07 & 91 & 2.95 & 56 & 2.30 & 2281 & 4.75 & 554 & 2.99 \\
\hline & NS & 1957 & 4.05 & 493 & 2.58 & 76 & 2.46 & 27 & 1.11 & 1899 & 3.96 & 470 & 2.54 \\
\hline & $\mathrm{IM}$ & 1888 & 3.91 & 428 & 2.24 & 50 & 1.62 & 23 & 0.94 & 1846 & 3.85 & 410 & 2.21 \\
\hline & RM & 1532 & 3.17 & 354 & 1.85 & 166 & 5.38 & 53 & 2.18 & 1408 & 2.93 & 323 & 1.74 \\
\hline & FM & 1656 & 3.43 & 220 & 1.15 & 110 & 3.57 & 26 & 1.07 & 1563 & 3.26 & 195 & 1.05 \\
\hline & $\mathrm{RD}$ & 258 & 0.53 & 295 & 1.54 & 1 & 0.03 & 1 & 0.04 & & & & \\
\hline & $E R$ & 101 & 0.21 & 451 & 2.36 & 1 & 0.03 & 4 & 0.16 & & & & \\
\hline & $\mathrm{GP}$ & 326 & 0.67 & 19 & 0.10 & 1 & 0.03 & 1 & 0.04 & & & & \\
\hline & Other $^{d}$ & 470 & 0.97 & 93 & 0.49 & & & & & & & & \\
\hline
\end{tabular}

$O A$ Osteoarthritis

${ }^{a} \mathrm{NHI}$, National Health Insurance; MD, Medicaid; VH, Veteran Healthcare

${ }^{\mathrm{b}} \mathrm{GH}$, General Hospital; TH, Tertiary Hospital; LCH, Long-term Care Hospital; PHC, Public Health Center; KMH, Korean Medicine Hospital

'OS, Orthopedic surgery; AN, Anesthesiology; GS, General Surgery; NS, Neurosurgery; IM, Internal Medicine; RM, Rehabilitation Medicine; FM, Family Medicine; RD, Radiology; EM, Emergency Medicine; GP, General Physician

dIncluding Neurology, Thoracic and Cardiovascular Surgery, Pediatrics, Obstetrics and Gynecology, Urology, and Neuropsychiatry

costs of visits (consultations), hospitalizations, medications, injections, anesthesia, physiotherapy, psychotherapy, procedures/surgeries, examinations, and radiologic evaluations/interventions. The categories reflecting the bulk of total expenses for each diagnostic group were, in decreasing order, procedures/surgeries, injections, visits (consultations), and hospitalizations for knee OA patients, and procedures/surgeries, hospitalizations, visits (consultations), and injections for knee meniscal and ligament injury cases. Procedures/surgeries constituted $\geq 30 \%$ of total expenses in both knee disorder categories, and injection costs comprised nearly $20 \%$ of the total expenses for knee OA patients, ranking 2nd in total costs, but only $6 \%$ of the total expenses for meniscal and ligament injury patients. More than $99 \%$ of all patients, irrespective of specific knee disorder, paid for cost of 
visits (consultations), and this category represented the third highest percentage of total costs for patients with either disorder. In addition, hospitalization costs also comprised a considerable portion of total medical expenses. In particular, hospitalization costs represented almost 20\% (second highest percentage) of the total expenses for knee meniscal and ligament injury patients. Although more than $13 \%$ of all patients were prescribed medications, total medication costs were $<2 \%$ of the total expenses for patients with either knee disorder. Similarly, $\geq 53 \%$ of all patients underwent physiotherapy, but this treatment cost represented only $6 \%$ of total expenses. The other subcategories (i.e., radiographic evaluations/interventions, examinations, and anesthesia) took up $<6 \%$ of all expenses, and psychotherapy was rarely performed. Despite its relatively low proportion out of total costs, radiographic evaluations/interventions were widely applied to $98 \%$ of knee OA patients and $94 \%$ of meniscal and ligament injury patients (Table 3).

\section{Usual care of knee $O A$ and knee meniscal and ligament injuries, excluding medications}

Frequently used surgeries, injections, and physiotherapies were investigated to determine usual practice patterns. Replacement arthroplasty was the most frequently performed surgery for knee OA patients, and cruciate ligament or meniscus surgery was the most common for knee meniscal and ligament injuries. Specifically, replacement arthroplasty usually involved total arthroplasty, and menisectomy was performed as either unilateral medial or unilateral lateral menisectomy. Patients who received surgical care were generally hospitalized.

Subcutaneous and intramuscular injections were performed in 19,722 knee OA cases and in 7390 meniscal and ligament injury cases, taking up approximately $40 \%$ of the cases of each disorder. In contrast, intra-articular injections were performed in 26,883 (55\%) knee OA cases and in 549 (3\%) meniscal and ligament injury cases. Upon hospitalization, subcutaneous and intramuscular injection rates increased to $83 \%$ and $65 \%$ for knee $\mathrm{OA}$ and meniscal and ligament injury patients, respectively, representing rates that were approximately twice those observed in outpatients. Intra-articular injections were administered to $55 \%$ of knee OA outpatients, but to only $14 \%$ of inpatients.

Of various physiotherapy modalities, superficial heat therapy, deep heat therapy, transcutaneous electrical nerve stimulation, and interferential current therapy were prescribed to about $25 \%$ of patients of either knee disorder; other physiotherapies were seldom used. Physiotherapy use was slightly more common for knee OA than meniscal and ligament injury patients. However, in hospitalized patients, exercise therapy was prescribed to $59 \%$ of knee OA and $27 \%$ of meniscal and ligament injury patients, which is significantly higher than the rates observed in ambulatory settings (Table 4).

\section{Medication use in usual care of knee $\mathrm{OA}$ and knee meniscal and ligament injuries}

The medications, including both narcotics and nonnarcotics, frequently used for knee disorders were organized according to 5th ATC levels. The most frequently used medication, for both knee OA and meniscal and ligament injury patients, was aceclofenac, which was administered to $20-30 \%$ of all knee disorder patients. Tramadol and diclofenac were also commonly prescribed, but their use was more pronounced in inpatients than in outpatients. In contrast, paracetamol was more frequently used among outpatients than among

Table 3 Distribution of medical expenditure in knee osteoarthritis and knee meniscal and ligament injury

\begin{tabular}{|c|c|c|c|c|c|c|c|c|c|c|}
\hline \multirow[t]{3}{*}{ Classification } & \multicolumn{5}{|l|}{ Knee OA } & \multicolumn{5}{|c|}{ Knee meniscal and ligament injury } \\
\hline & \multicolumn{2}{|l|}{ Case (n) } & \multicolumn{2}{|l|}{$\operatorname{Cost}^{a}$} & \multirow[t]{2}{*}{ Per-case cost ${ }^{a}$} & \multicolumn{2}{|l|}{ Case (n) } & \multicolumn{2}{|l|}{$\operatorname{Cost}^{a}$} & \multirow[t]{2}{*}{ Per-case cost ${ }^{a}$} \\
\hline & $N=48,321$ & $\%$ & Total & $\%$ & & $N=19,136$ & $\%$ & Total & $\%$ & \\
\hline Procedure/surgery & 5403 & 11.18 & $7,593,254,872$ & 31.41 & $1,405,377.5$ & 4555 & 23.80 & $1,887,655,557$ & 34.31 & $414,414.0$ \\
\hline Injection & 37,050 & 76.67 & $4,732,291,088$ & 19.58 & $127,727.2$ & 8315 & 43.45 & $365,245,211$ & 6.64 & $43,926.1$ \\
\hline Outpatient visit (consultation) & 48,283 & 99.92 & $4,021,230,425$ & 16.64 & $83,284.6$ & 19,084 & 99.73 & $715,896,835$ & 13.01 & $37,512.9$ \\
\hline Hospitalization & 3033 & 6.28 & $2,757,254,594$ & 11.41 & $909,084.9$ & 2403 & 12.56 & $1,186,629,123$ & 21.57 & $493,811.5$ \\
\hline Physiotherapy & 25,667 & 53.12 & $1,337,965,293$ & 5.54 & $52,127.8$ & 11,048 & 57.73 & $334,564,005$ & 6.08 & $30,282.8$ \\
\hline $\begin{array}{l}\text { Radiographic evaluation/ } \\
\text { intervention }\end{array}$ & 47,232 & 97.75 & $1,277,960,897$ & 5.29 & $27,057.1$ & 17,919 & 93.64 & $323,895,267$ & 5.89 & $18,075.5$ \\
\hline Examination & 10,310 & 21.34 & $1,144,231,241$ & 4.73 & $110,982.7$ & 3351 & 17.51 & $357,995,050$ & 6.51 & $106,832.3$ \\
\hline Anesthesia & 8576 & 17.75 & $907,924,433$ & 3.76 & $105,868.1$ & 1829 & 9.56 & $224,006,652$ & 4.07 & $122,474.9$ \\
\hline Medication & 6327 & 13.09 & $399,489,786$ & 1.65 & $63,140.5$ & 3043 & 15.90 & $105,707,519$ & 1.92 & $34,737.9$ \\
\hline Psychotherapy & 21 & 0.04 & 674,087 & 0.00 & $32,099.4$ & 1 & 0.01 & 12,579 & 0.00 & $12,579.0$ \\
\hline
\end{tabular}

OA Osteoarthritis

a Displayed in KRW; 1 USD = 1104 KRW (as of September 30th, 2016) 


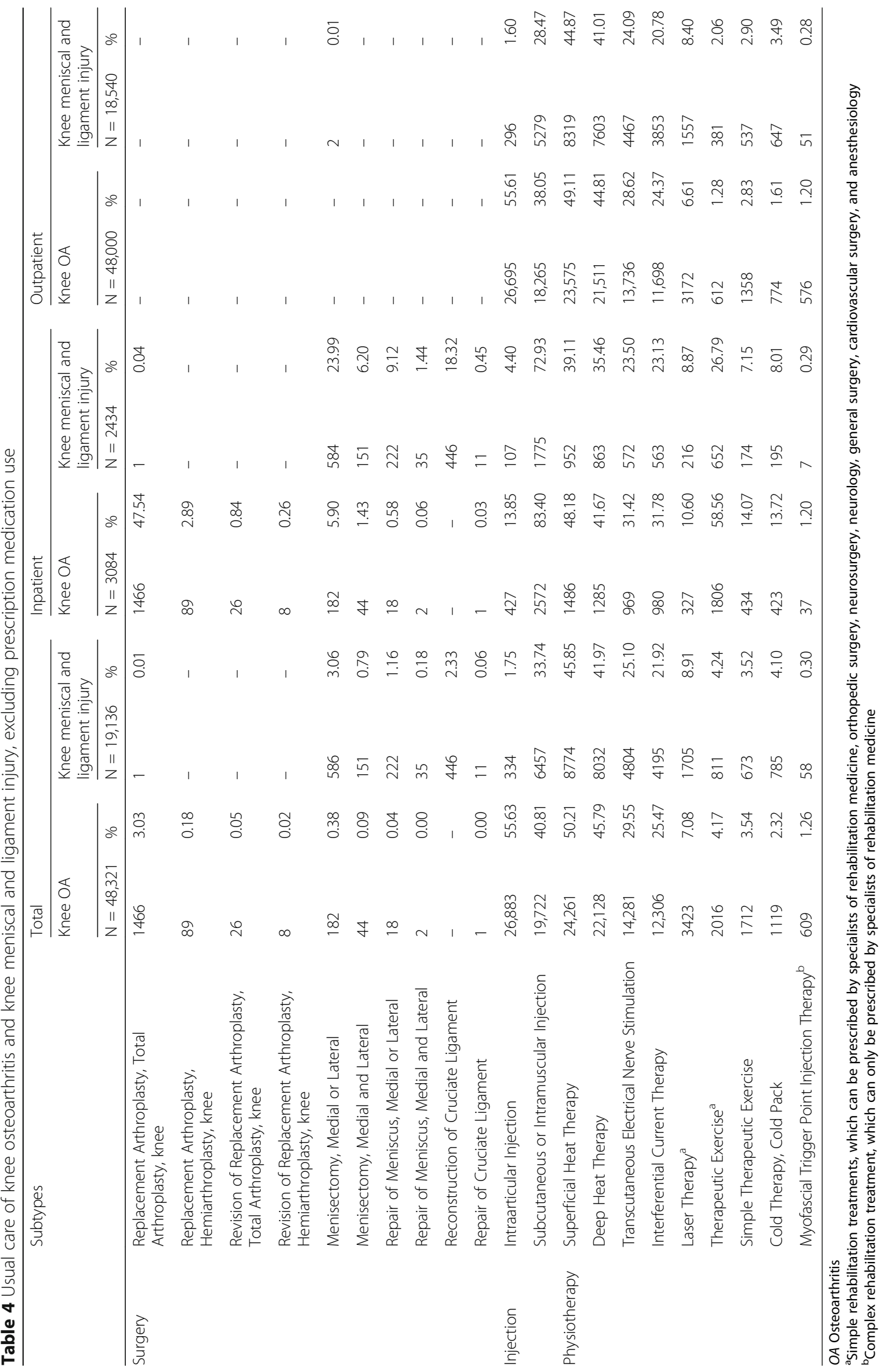


inpatients. Meloxicam and celecoxib were also largely used for knee OA patients, but their use was not evident in knee meniscal and ligament injury patients. Pethidine was the most commonly used narcotic analgesic, with $25 \%$ of knee OA inpatients receiving pethidine, although the overall prescription rate was low (Table 5).

\section{Discussion}

The total expenses, per-patient expenses, average days of care, and average number of visits were higher for inpatients than for outpatients, regardless of the specific type of knee disorder diagnosis (knee OA or meniscal and ligament injury). These results may be partly attributed to differences in disease and symptom severity as patients with more severe disease and/or symptoms may be more likely to undergo hospitalized care. Another possible explanation is that surgical interventions are more often performed in inpatient settings (Table 4). The current results support the view that surgical interventions take up a substantial portion of knee disorderrelated costs. The incidence of knee OA was highest among females aged $\geq 50$ years, which is consistent with a previous report by Arden et al. [20]. Conversely, knee meniscal and ligament injuries occurred more often among those aged $<60$ years. Although the incidence of these injuries was highest among children and adolescents, hospitalized patients amounted to only half of the outpatient sector in this age group, suggesting that physically active younger individuals may be more prone to injury but injuries may be milder.

The costs for procedures/surgeries, injections, visits (consultations), and hospitalizations comprised $\geq 75 \%$ of the total expenses for patients with either type of knee disorder. Although the large number of injection treatments is mainly responsible for this finding, it is worth note that the number of surgery and hospitalization cases was relatively small compared to the total costs. It can be carefully inferred that performing surgical operations in inpatient settings is likely to drastically increase per-patient expenses. Injection treatments constituted the second largest expense in knee OA patients, and hospitalization was the 2nd largest expense for knee meniscal and ligament injury patients. To ease the socioeconomic burden of these disorders (i.e., total expenses and per-patient expenses), studies should be conducted, from the policymakers' perspective, on appropriate practice guidelines and adequate compensation costs, especially regarding surgical interventions, injection treatments, and hospitalization.

The types of surgical interventions performed in the two diagnostic groups were highly disparate, with

Table 5 Medication prescribed for knee osteoarthritis and knee meniscal and ligament injury as assessed at the 5th Anatomical Therapeutic Chemical Classification System level

\begin{tabular}{|c|c|c|c|c|c|c|c|c|c|c|c|c|}
\hline \multirow[t]{3}{*}{ 5th ATC level } & \multicolumn{4}{|l|}{ Total } & \multicolumn{4}{|l|}{ Inpatient } & \multicolumn{4}{|l|}{ Outpatient } \\
\hline & \multicolumn{2}{|l|}{ Knee OA } & \multicolumn{2}{|c|}{$\begin{array}{l}\text { Knee meniscal and } \\
\text { ligament injury }\end{array}$} & \multicolumn{2}{|l|}{ Knee OA } & \multicolumn{2}{|c|}{$\begin{array}{l}\text { Knee meniscal and } \\
\text { ligament injury }\end{array}$} & \multicolumn{2}{|l|}{ Knee OA } & \multicolumn{2}{|c|}{$\begin{array}{l}\text { Knee meniscal and } \\
\text { ligament injury }\end{array}$} \\
\hline & $N=48,321$ & $\%$ & $N=19,136$ & $\%$ & $N=3084$ & $\%$ & $N=2434$ & $\%$ & $N=48,000$ & $\%$ & $N=18,540$ & $\%$ \\
\hline Aceclofenac & 15,810 & 32.72 & 4961 & 25.92 & 840 & 27.24 & 858 & 35.25 & 15,357 & 31.99 & 4499 & 24.27 \\
\hline Tramadol & 10,651 & 22.04 & 2982 & 15.58 & 1743 & 56.52 & 837 & 34.39 & 9364 & 19.51 & 2291 & 12.36 \\
\hline Diclofenac & 9068 & 18.77 & 3688 & 19.27 & 1764 & 57.20 & 1132 & 46.51 & 7734 & 16.11 & 2832 & 15.28 \\
\hline Meloxicam & 10,785 & 22.32 & 597 & 3.12 & 524 & 16.99 & 178 & 7.31 & 10,544 & 21.97 & 486 & 2.62 \\
\hline Loxoprofen sodium hydrate ${ }^{a}$ & 5598 & 11.59 & 3295 & 17.22 & 291 & 9.44 & 358 & 14.71 & 5375 & 11.20 & 3043 & 16.41 \\
\hline Tramadol, combinations & 7082 & 14.66 & 1588 & 8.30 & 582 & 18.87 & 248 & 10.19 & 6741 & 14.04 & 1423 & 7.68 \\
\hline Talniflumate ${ }^{a}$ & 5273 & 10.91 & 3127 & 16.34 & 432 & 14.01 & 519 & 21.32 & 4944 & 10.30 & 2747 & 14.82 \\
\hline Paracetamol & 4988 & 10.32 & 1435 & 7.50 & 843 & 27.33 & 364 & 14.95 & 4299 & 8.96 & 1133 & 6.11 \\
\hline Celecoxib & 4791 & 9.91 & 134 & 0.70 & 634 & 20.56 & 43 & 1.77 & 4490 & 9.35 & 106 & 0.57 \\
\hline Piroxicam & 2085 & 4.31 & 307 & 1.60 & 220 & 7.13 & 116 & 4.77 & 1908 & 3.98 & 200 & 1.08 \\
\hline Zaltoprofen $^{a}$ & 1531 & 3.17 & 753 & 3.93 & 138 & 4.47 & 113 & 4.64 & 1432 & 2.98 & 666 & 3.59 \\
\hline Chlorphenesin carbamate $^{\mathrm{a}}$ & 1597 & 3.30 & 599 & 3.13 & 69 & 2.24 & 46 & 1.89 & 1565 & 3.26 & 568 & 3.06 \\
\hline Dexibuprofen & 1105 & 2.29 & 509 & 2.66 & 36 & 1.17 & 23 & 0.94 & 1072 & 2.23 & 488 & 2.63 \\
\hline Ketorolac & 777 & 1.61 & 279 & 1.46 & 633 & 20.53 & 230 & 9.45 & 152 & 0.32 & 49 & 0.26 \\
\hline Nabumetone & 904 & 1.87 & 114 & 0.60 & 46 & 1.49 & 37 & 1.52 & 873 & 1.82 & 83 & 0.45 \\
\hline Pethidine $^{b}$ & 858 & 1.78 & 151 & 0.79 & 843 & 27.33 & 145 & 5.96 & 18 & 0.04 & 7 & 0.04 \\
\hline
\end{tabular}

ATC Anatomical Therapeutic Chemical, OA Osteoarthritis

${ }^{a}$ Chemical name of medicine with no corresponding 5th level ATC codes

${ }^{\mathrm{b}}$ Narcotics; otherwise, non-narcotics 
replacement arthroplasty being performed most frequently in cases of knee OA, and meniscal and ligament operations (i.e., injured menisci or ligaments) being most common in cases of knee meniscal and ligament injury. Subcutaneous and intramuscular injection treatments were conducted in 19,722 knee OA cases and in 7390 knee meniscal and ligament injury cases, each comprising about $40 \%$ of all cases. However, the percentages of patients receiving subcutaneous and intramuscular injections rose to $83 \%$ in knee OA inpatients and to $65 \%$ in meniscal and ligament injury inpatients. Although intraarticular injections were frequently employed for knee OA outpatients, they were seldom used in patients with knee meniscal and ligament injury.

Heat therapy and electrostimulation are two types of physiotherapy that may be considered typical care for knee OA and meniscal and ligament injuries. More than $58 \%$ of hospitalized knee OA patients were prescribed therapeutic exercise, which was more common than superficial heat therapy. Therapeutic exercise is speculated to be mainly used as a means of post-surgical rehabilitation. Laser therapy and therapeutic exercise were considered simple rehabilitation treatment methods; myofascial trigger point injection, a complex rehabilitation method; and the other subtypes to be basic physiotherapies. Basic physiotherapy can be prescribed by all physicians without restriction as opposed to simple and complex rehabilitation therapies which can only be prescribed by relevant specialists. Therefore, limitations in the ability to prescribe laser therapy, therapeutic exercise, and myofascial trigger point injection treatments should be considered when interpreting these results.

Diclofenac is a non-steroidal anti-inflammatory drug (NSAID) that is widely used as first-line therapy for chronic inflammatory states including OA. However, prolonged use of diclofenac has been associated with gastrointestinal adverse events such as bleeding, ulcers, and perforations in severe cases. Aceclofenac, which has a similar chemical structure, was developed to address such complications and has been proven to be a safer alternative [21]. Aceclofenac was shown to be used more frequently than diclofenac in this study, possibly because it exhibits fewer side effects. Diclofenac tended to be more frequently administered in inpatient settings where adverse event monitoring is easier.

Tramadol is an opioid-like analgesic that affects the central nervous system (CNS) [22]. Tramadol, a $\mu$ opioid receptor agonist, exerts nociceptive effects by increasing serotonin and noradrenaline levels in the CNS [23]. As the mechanisms of action differ between tramadol and NSAIDs, tramadol can be effectively used in patients with pain not responding adequately to NSAID treatment [24]. The present study results revealed that tramadol was the second most commonly prescribed drug. Moreover, tramadol combinations were the sixth most common prescription, under a separate ATC code, and taken together, the number of tramadol prescriptions was nearly as high as those for aceclofenac.

Paracetamol, also known as acetaminophen, is recommended as a first-line analgesic for knee OA patients by the European League against Rheumatism, American College of Rheumatology (ACR), and Osteoarthritis Research Society International. Long-term use of paracetamol is also preferred over other medications [25]. Although the analgesic effect of paracetamol is relatively weak compared to that of NSAIDs or COX-2 selective inhibitors, it is widely used as it is better tolerated [26]. However, paracetamol is often implicated in druginduced liver injuries with approximately 30,000 patients being hospitalized annually for paracetamol-related liver injuries in the United States [27]. Nevertheless, paracetamol hepatotoxicity has been reported to be minimized by avoiding overdosing $[28,29]$.

One strength of classifying drugs according to 5th ATC levels is that complete identification of specific chemicals is possible, while limitations include the fact that identification and recognition of prescription patterns is not easy. The most frequently prescribed drug substances are listed in Table 5. Complete listings of prescribed drugs are organized by non-narcotics and narcotics in Additional file 3: Table S3 and Additional file 4: Table S4. At the 4th ATC level, the non-narcotic and narcotic drugs consisted of 18 and 4 subgroups, respectively (Additional file 5: Table S5). In order of prescription frequency, acetic acid derivatives and related substances, other opioids, propionic acid derivatives, oxicams, other anti-inflammatory and anti-rheumatic agents, and non-steroids were the most frequently used drugs in the non-narcotic analgesic subgroups, and phenylpiperidine derivatives, natural opium alkaloids, opioid anesthetics, and opium alkaloids and their derivatives were the most frequently prescribed drugs from the narcotic analgesic subgroups.

Investigations of medical service use at a national level hold heightened significance in that various disparities exist between evidence-based medicine and the actual practice selected for knee disorders in real world settings. For example, while use of meniscectomy is similarly high in Korea as in the U.S., a recent high-quality $\mathrm{RCT}$ reported that its effects did not surpass that of sham controls [30]. Similarly, although total knee replacement (TKR) is the most common surgical intervention for knee OA in Korea and is performed in 670,000 new cases in the U.S. annually, comparison of TKR and 12 weeks of conservative treatment found that while TKR resulted in significant differences in pain reduction, it also entailed serious complications, and most of the conservative treatment group exhibited meaningful 
improvement without surgery [31]. Moreover, intraarticular triamcinolone injections, which are commonly used in knee OA patients, failed to provide pain relief over saline, and were associated with a significantly larger decrease in joint cartilage [32].

Study limitations include the following: While the study analyses were based on codes filed in the national claims database, the level of accuracy of the diagnosing process itself could not be verified and is beyond the scope of this study. For example, a physician may have reached a diagnosis not conforming to such wellestablished diagnostic standards as proposed by the ACR [33]. In addition, outpatient medication intake could only be assessed from database prescription records rather than actual medication intake records. Regarding terminology, although the term incidence was used throughout the manuscript to indicate new cases within the index period, readers should take into account that the incidence was limited to new symptomatic cases that received diagnosis and treatment through visits to medical institutions for medical service use. Moreover, the current database does not contain information regarding treatments and medications not covered by National Health Insurance or over-the-counter medication. While other studies using claims data carry similar limitations, these uncertainties need to be given due consideration when interpreting these results.

Records of medical services, including surgeries, injections, physiotherapy, and analgesic use, could not be solely attributed to knee disorders if the patient had coexisting conditions and was coded with different subsets of disease codes. Although codes that were clearly non-knee-associated were excluded, such consolidations may be an additional limitation of this study, as it may have introduced unintentional selection bias. Also of note is that both conventional and traditional Korean medicine (TKM) are recognized by Korean medical and legal regulatory bodies in a dual medical system and are covered by National Health Insurance. Many patients seek acupuncture and pharmacopuncture for treatment of musculoskeletal disorders. In a previous report on TKM care and low back pain in Korea using 2011 HIRA-NPS data, TKM use was highest $(28.8 \%)$ in patients with low back pain [34]. Unfortunately, the 2014 dataset currently available to the public does not include records on TKM use, limiting the present study results to conventional medicine services, and precluding a more comprehensive and complete assessment of knee disorder treatments in Korea.

\section{Conclusions}

This study analyzed HIRA-NPS data to investigate the current incidence of knee disorders and clinical practice patterns for knee $\mathrm{OA}$ and knee meniscal and ligament injuries. The medical expense analysis (i.e., amounts and distribution) may provide further information to policymakers. Further, the detailed description of injection, physiotherapy, and analgesic use may prove valuable for researchers and practitioners seeking to understand the constituents of usual care in actual clinical practice. Additional research is warranted, particularly regarding treatment codes (e.g., M22 and M23) not covered in the present study. Also, the HIRA-NPS dataset only contains billing records for a single calendar year, rendering longitudinal studies with time series analyses of natural history and causal relationships impossible; national cohort data extracted from national health insurance claims data [35] may be analyzed for such purposes in future studies.

\section{Additional files}

Additional file 1: Table S1. Diagnostic codes of knee disorders following the Korean Standard Classification of Diseases, 6th revision (KCD-6) adapted from the International Classification of Diseases, 10th revision. (ICD-10) (DOCX 20 kb)

Additional file 2: Table S2. Definition of medical care for knee disorders from given codes. (DOCX $18 \mathrm{~kb}$ )

Additional file 3: Table S3. Non-narcotic medications in knee osteoarthritis and knee meniscal and ligament injury as assessed at the 5th Anatomical Therapeutic Chemical Classification System level. (DOCX 30 kb)

Additional file 4: Table S4. Narcotic medications in knee osteoarthritis and knee meniscal and ligament injury as assessed at the 5th Anatomical Therapeutic Chemical Classification System level. (DOCX 19 kb)

Additional file 5: Table S5. Total medications in knee osteoarthritis and knee meniscal and ligament injury as assessed at the 4th Anatomical Therapeutic Chemical Classification System level. (DOCX 23 kb)

\section{Abbreviations}

ACL: Anterior cruciate ligament; ACR: American College of Rheumatology; ATC: Anatomical Therapeutic Chemical; CNS: Central nervous system; COX2: Cyclooxygenase-2; EM: Emergency medicine; HIRA: Korean Health Insurance Review and Assessment Service; ICD: International Classification of Diseases; NHIS: National Health Insurance Service; NPS: National Patient Sample; NSAID: Non-steroidal anti-inflammatory drug; OA: Osteoarthritis; TKM: Traditional Korean medicine; TKR: Total knee replacement

\section{Acknowledgements}

Not applicable.

\section{Funding}

This research was supported by the Traditional Korean Medicine R\&D program funded by the Ministry of Health \& Welfare through the Korea Health Industry Development Institute (KHIDI) (HI17C0761).

\section{Availability of data and materials}

The HIRA-NPS dataset that supports the findings of this study is made available from HIRA but restrictions apply to data availability from the authors as it was used under license.

\section{Authors' contributions}

CYS, YJL, MRK, and IHH conceptualized and designed the study. JSS and $J \mathrm{~L}$ acquired the data. CYS, WK, YYC, BCS, EHH, KS and MK analyzed and interpreted the data. CYS, JSS, MRK, and WK drafted the manuscript. JL, YJL, YYC, BCS, EHH, KS, MK, and $I H H$ revised the manuscript critically for important intellectual content. YJL acquired funding. All authors have read and approved the final manuscript. 


\section{Ethics approval and consent to participate}

The current study was reviewed and approved by the Institutional Review Board of Jaseng Hospital of Korean Medicine (JASENG 2016-10-004). The current study was performed in accordance with the ethical standards of the 1964 Declaration of Helsinki and the U.S. Health Insurance Portability and Accountability Act (HIPAA). Written informed consent was not obtained from participants as the sample dataset used in this study was extracted from Korean National Health Insurance claims data and is made publicly available for research purposes by the Health Insurance Review and Assessment Service (HIRA). All data are removed of identifying personal information by HIRA prior to analysis and therefore participants remained anonymous.

\section{Consent for publication}

Not applicable.

\section{Competing interests}

The authors declare that they have no competing interests.

\section{Publisher's Note}

Springer Nature remains neutral with regard to jurisdictional claims in published maps and institutional affiliations.

\section{Author details}

'Jaseng Spine and Joint Research Institute, Jaseng Medical Foundation, 858 Eonju-ro, Gangnam-gu, Seoul, Republic of Korea. ${ }^{2}$ Department of Applied Korean Medicine, College of Korean Medicine, Graduate School, Kyung Hee University, Dongdaemun-gu, Seoul, Republic of Korea. ${ }^{3}$ Department of Rehabilitation Medicine of Korean Medicine, College of Korean Medicine, Sangji University, Wonju-si, Gangwon-do, Republic of Korea. ${ }^{4}$ Spine \& Joint Center, Pusan National University Korean Medicine Hospital, Yangsan-si, Gyeongsangnam-do, Republic of Korea. ${ }^{5}$ Department of Korean Rehabilitation Medicine, School of Korean Medicine, Pusan National University, Yangsan-si, Gyeongsangnam-do, Republic of Korea. ${ }^{6}$ Prevention Sciences, Rollins School of Public Health, Emory University, Atlanta, GA, USA. ${ }^{7}$ Department of Cardiovascular and Neurological Diseases (Stroke Center), College of Korean Medicine, Kyung Hee University, Seoul, Republic of Korea.

Received: 11 April 2017 Accepted: 31 October 2017

Published online: 10 November 2017

\section{References}

1. Zhang Y, Jordan JM. Epidemiology of osteoarthritis. Clin Geriatr Med. 2010; 26(3):355-69.

2. Park J-H, Hong J-Y, Han K, Suh S-W, Park S-Y, Yang J-H, et al. Prevalence of symptomatic hip, knee, and spine osteoarthritis nationwide health survey analysis of an elderly Korean population. Medicine. 2017;96(12)

3. Jordan MR. Lateral meniscal variants: evaluation and treatment. J Am Acad Orthop Surg. 1996:4(4):191-200.

4. Kim S-J, Lee Y-T, Kim D-W. Intraarticular anatomic variants associated with discoid meniscus in Koreans. Clin Orthop Relat Res. 1998;356:202-7.

5. Kotlarz H, Gunnarsson CL, Fang H, Rizzo JA. Osteoarthritis and absenteeism costs: evidence from US National Survey Data. J Occup Environ Med. 2010; 52(3):263-8.

6. Cullen KA, Hall MJ, Golosinskiy A. Ambulatory surgery in the United States, 2006. Natl Health Stat Report. 2009;(11):1-25.

7. Peat G, Bergknut C, Frobell R, Jöud A, Englund M. Population-wide incidence estimates for soft tissue knee injuries presenting to healthcare in southern Sweden: data from the Skåne healthcare register. Arthritis research \& therapy. 2014:16(4):R162.

8. Kujala UM, Taimela S, Viljanen T. Leisure physical activity and various pain symptoms among adolescents. Br J Sports Med. 1999;33(5):325-8.

9. Ding C, Cicuttini F, Jones G. Tibial subchondral bone size and knee cartilage defects: relevance to knee osteoarthritis. Osteoarthr Cartil. 2007:15(5):479-86.

10. Yelin E, Callahan LF. Special article the economic cost and social and psychological impact of musculoskeletal conditions. Arthritis. Rheumatology. 1995;38(10):1351-62.

11. Muthuri S, McWilliams D, Doherty M, Zhang W. History of knee injuries and knee osteoarthritis: a meta-analysis of observational studies. Osteoarthr Cartil. 2011;19(11):1286-93.

12. Kim L, Kim J-A, Kim SA. Guide for the utilization of health insurance review and assessment service national patient samples. Epidemiology and health. 2014;36
13. Bergkvist D, Dahlberg LE, Neuman P, Englund M. Knee arthroscopies: who gets them, what does the radiologist report, and what does the surgeon find? An evaluation from southern Sweden. Acta Orthop. 2016;87(1):12-6.

14. Prieto-Alhambra D, Judge A, Javaid MK, Cooper C, Diez-Perez A, Arden NK. Incidence and risk factors for clinically diagnosed knee, hip and hand osteoarthritis: influences of age, gender and osteoarthritis affecting other joints. Ann Rheum Dis. 2014;73(9):1659-64.

15. Hubertsson J, Petersson IF, Thorstensson CA, Englund M. Risk of sick leave and disability pension in working-age women and men with knee osteoarthritis. Ann Rheum Dis. 2013;72(3):401-5.

16. Peat G, Bergknut C, Frobell R, Joud A, Englund M. Population-wide incidence estimates for soft tissue knee injuries presenting to healthcare in southern Sweden: data from the Skane healthcare register. Arthritis Res Ther. 2014;16(4):R162.

17. Lee SM, Han SK, Kim JH, Jang BH, Cheong $\mathrm{CL}$, Son HJ, et al. Clinical effectiveness of injection therapy for chronic low back pain. In: Korean national evidence-based health care collaborating agency; 2010. p. 1-232.

18. ATC/DDD Methodology: History [http://www.whocc.no/atc_ddd_ methodology/history].

19. ATC: Structure and principles [http://www.whocc.no/atc/structure_and_ principles].

20. Arden N, Nevitt MC. Osteoarthritis: epidemiology. Best Pract Res Clin Rheumatol. 2006;20(1):3-25.

21. Sharma G, Singh J, Anand D, Kumar M, Raza K, Pareek A, et al. Aceclofenac: species-dependent metabolism and newer paradigm shift from oral to nonoral delivery. Curr Top Med Chem. 2016;

22. Merashly M, Uthman I. Management of knee osteoarthritis: an evidencebased review of treatment options. J Med Liban. 2012;60(4):237-42.

23. Desmeules JA. The tramadol option. Eur J Pain. 2000; 4 Suppl A:15-21.

24. Inage K, Orita S, Yamauchi K, Suzuki T, Suzuki M, Sakuma Y, et al. Low-dose tramadol and non-steroidal anti-inflammatory drug combination therapy prevents the transition to chronic low back pain. Asian. Spine J. 2016;10(4):685-9.

25. American Geriatrics Society Panel on the Pharmacological Management of Persistent Pain in Older. P. Pharmacological management of persistent pain in older persons. Pain Med. 2009;10(6):1062-83.

26. Graham GG, Davies MJ, Day RO, Mohamudally A, Scott KF. The modern pharmacology of paracetamol: therapeutic actions, mechanism of action, metabolism, toxicity and recent pharmacological findings. Inflammopharmacology. 2013;21(3):201-32.

27. Blieden M, Paramore LC, Shah D, Ben-Joseph RA. Perspective on the epidemiology of acetaminophen exposure and toxicity in the United States. Expert Rev Clin Pharmacol. 2014;7(3):341-8.

28. Sinatra RS, Jahr JS, Reynolds LW, Viscusi ER, Groudine SB, Payen-Champenois C. Efficacy and safety of single and repeated administration of 1 gram intravenous acetaminophen injection (paracetamol) for pain management after major orthopedic surgery. Anesthesiology. 2005;102(4):822-31.

29. Clark R, Fisher JE, Sketris IS, Johnston GM. Population prevalence of high dose paracetamol in dispensed paracetamol/opioid prescription combinations: an observational study. BMC Clin Pharmacol. 2012;12:11.

30. Sihvonen R, Paavola M, Malmivaara A, Itälä A, Joukainen A, Nurmi $\mathrm{H}$, et al. Arthroscopic partial meniscectomy versus sham surgery for a degenerative meniscal tear. N Engl J Med. 2013;369(26):2515-24.

31. Skou ST, Roos EM, Laursen MB, Rathleff MS, Arendt-Nielsen L, Simonsen O, et al. A randomized, controlled trial of total knee replacement. N Engl J Med. 2015;373(17):1597-606.

32. McAlindon TE, LaValley MP, Harvey WF, Price LL, Driban JB, Zhang M, et al. Effect of intra-articular triamcinolone vs saline on knee cartilage volume and pain in patients with knee osteoarthritis: a randomized clinical trial. JAMA. 2017:317(19):1967-75.

33. Hochberg MC, Altman RD, Brandt KD, Clark BM, Dieppe PA, Griffin MR, et al. Guidelines for the medical management of osteoarthritis. Part II. Osteoarthritis of the knee. American College of Rheumatology. Arthritis Rheum. 1995;38(11):1541-6.

34. Ahn YJ, Shin JS, Lee J, Lee YJ, Kim MR, Park KB, et al. Evaluation of use and cost of medical care of common lumbar disorders in Korea: cross-sectional study of Korean Health Insurance Review and Assessment Service National Patient Sample data. BMJ Open. 2016;6(9):e012432.

35. Specifics of National Cohort Sample database [https://nhiss.nhis.or.kr/bd/ab/ bdaba002cr.do]. 